\title{
Microrriego de brinzales a través de recipientes porosos enterrados: Fundamentos teóricos y prácticos
}

\author{
Martínez de Azagra Paredes, A. ${ }^{* 1}$, Del Río San José, J. ${ }^{2}$ \\ ${ }^{1}$ Unidad Docente de Hidráulica e Hidrología Forestal. E.T.S. de Ingenierías Agrarias. U. de Valladolid. \\ Avenida de Madrid, 44. 34004 Palencia. \\ ${ }^{2} J u n t a$ de Castilla y León. Delegación Territorial de Valladolid. Servicio Territorial de Medio Ambiente. \\ Duque de la Victoria, 8. 47001 Valladolid. \\ e-mail: amap@iaf.uva.es
}

\section{Resumen}

Tras una breve puesta al día sobre estos sistemas de riego, se define el concepto de rezumador y se determina su ecuación característica. A partir de esta ecuación y del potencial hídrico de referencia, se obtiene el tiempo de vaciado del recipiente poroso, parámetro básico para el diseño y posterior manejo de estos sistemas de riego. Se comentan las ventajas, inconvenientes y limitaciones del riego a través de paredes porosas (rezumadores).

Palabras clave: emisor de riego no convencional, evitación de marras, oasificación, repoblación forestal, riegos de apoyo, zonas áridas.

\section{Introducción}

Los microrriegos iniciales de arraigo (al plantar) así como los de apoyo y de socorro para el establecimiento de árboles (durante el o los primeros años) son muy convenientes para reducir las marras por estrés hídrico (Bainbridge, 2007). Tal ocurre en zonas áridas, subdesérticas y desérticas (con lluvias escasas, esporádicas e inciertas) y en áreas críticas para la vegetación (secarrales; solanas; lugares con suelos muy someros, muy arenosos, salinos o yesosos; zonas muy ventosas; escombreras; huecos de minería, etc.).

Se trata de una estrategia muy útil para restaurar (es decir: para oasificar) espacios degradados en donde apenas crezca vegetación. Estos riegos permiten ampliar 
el periodo de plantación o de siembra y resultan complementarios a las técnicas tradicionales de recolección de agua (Navarro et al., 2009). Frente a las cosechas de agua tienen como principal ventaja su independencia de las siempre aleatorias lluvias. A cambio, requieren de alguna fuente de agua segura y son más costosos.

Aunque pueden utilizarse sistemas de riego convencionales (aspersión, goteo, o gravedad en alcorques), muchas veces se usan métodos no convencionales: riego a través de tubos verticales hincados en el suelo, riego a través de tubos horizontales de drenaje, o a través de mechas, o mediante superficies porosas o con destiladores solares (Martínez de Azagra y Del Río, 2012).

Estos sistemas específicos de microrriego difieren de los practicados en agricultura por dos motivos principales: su objetivo y su necesidad de ahorrar agua. Los riegos de brinzales no persiguen una alta producción sino el establecimiento de una vegetación leñosa: árboles y/o arbustos bien adaptados al lugar que -una vez arraigados- prosperen y se desarrollen de manera autónoma, restaurando el ecosistema y/o mejorando el paisaje. Además y por estar pensados para zonas áridas, la eficiencia en la aplicación del agua resulta crucial.

Dentro de los métodos no convencionales citados, el riego a través de recipientes porosos, enterrados junto a las raíces de las plantas, merece aquí nuestra atención. Es un sistema muy antiguo ya que el hombre lo lleva practicando desde hace más de dos mil años. Pero es muy actual por su alta eficiencia en la aplicación de agua y por el renovado interés que su desarrollo despierta.

En el libro de Fan Shengzi (redactado en Shandong, China, en el siglo I antes de Cristo) se describe este sistema para cultivar melones (Sheng-Han, 1974; Bainbridge, 2001). A su vez, Tilló (2006) refiere que los fenicios enterraban vasijas de arci1la porosa al pie de los olivos y que las rellenaban con agua a intervalos regulares. También los romanos parecen haber conocido y difundido este sistema de irrigación (UNEP, 1997). Ello puede explicar el hecho de que en la cultura rural mediterránea el sistema sea bien conocido y que se aplique para instalar árboles frutales de secano (olivos, almendros, algarrobos, jerbos, etc.). En el sector forestal español también se aplica el método y recibe el expresivo nombre de repoblaciones con biberón (Serrada et al., 2005). En otros países, tan distantes como Pakistán o Cuba, el sector forestal también acude al método ante situaciones de repoblación difícil (Sheikh and Shah, 1983; Ortega et al., 1986).

Los primeros estudios sistemáticos sobre riegos a través de vasijas y cápsulas de barro se deben a Mondal (1974a, 1974b) en India y a Olguín $(1975,1977)$ en México, respectivamente. Después de estos trabajos pioneros se han sucedido numerosas investigaciones y ensayos sobre la materia que escapan de los objetivos de esta introducción. Destacan los trabajos de Stein $(1994,1998)$ en el ámbito agrícola y de Bainbridge $(2001,2002,2007)$ en el ámbito ecológico y forestal.

La posibilidad de sustituir el barro cocido por materiales plásticos porosos también ha sido ensayada. La propuesta de Matorel $(1996,1998)$ en Piura (Perú) es la primera de que tenemos noticia. La más reciente que hemos localizado es el sistema Moistube Microreservoir ${ }^{\circledR}$, desarrollado en 2012.

Dado que en ninguno de los artículos consultados se desarrolla el fundamento te- 
órico de estos sistemas de riego, en esta comunicación vamos a tratar de paliar esta carencia.

\section{Definición de rezumador}

Un rezumador es una pared rígida, porosa y saturada de agua que resuda lentamente en régimen laminar (número de Reynolds bajo, $\boldsymbol{R} \boldsymbol{e}<1$ ), humedeciendo de forma localizada el suelo circundante, sin necesidad de presión en la red o en la botella donde se encuentre inserto. El aporte de agua se produce conforme a la ley de Darcy (1856). Los rezumadores pueden ser de barro cocido o de materiales plásticos. Su superficie rezumante $(\boldsymbol{A})$ puede ocupar todo el área exterior del recipiente o sólo una parte del mismo (Fig. 1).

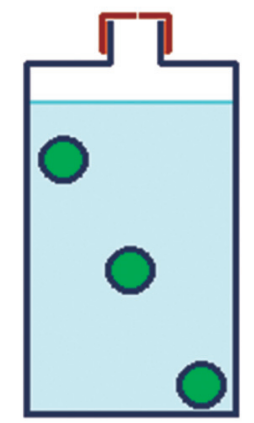

Figura 1. Recipiente con tres rezumadores circulares a distinto nivel.

\section{Ecuación característica de un rezumador}

La ecuación (o curva) característica de un rezumador es función de cuatro parámetros: el área del rezumador $\left(\boldsymbol{A}, \mathrm{en}^{2}\right)$, el espesor de su pared $(\boldsymbol{e}$, en $\mathrm{m})$, su permeabilidad $\left(\boldsymbol{k}\right.$ s, en $\left.\mathrm{m} \cdot \mathrm{s}^{-1}\right)$ y la diferencia de potencial hídrico $(\psi$, en $\mathrm{m})$ existente a un lado y otro del rezumador. Su expresión se deja obtener por integración, combinando la ecuación de Darcy con la ecuación de continuidad y fijando las condiciones de frontera.

La ley o ecuación de Darcy puede expresarse de la siguiente manera:

$$
q=v \cdot A=k_{s} \cdot \frac{H_{1}-H_{2}}{e} \cdot A
$$

siendo: $\boldsymbol{q}$ el caudal volumétrico $\left(\mathrm{en}^{3} \cdot \mathrm{s}^{-1}\right)$ que circula por el medio poroso en régimen laminar, $\boldsymbol{v}$ la velocidad de flujo $\left(\mathrm{en} \mathrm{m} \cdot \mathrm{s}^{-1}\right), \boldsymbol{A}$ la sección transversal atravesada por el flujo, $\boldsymbol{k}_{\mathrm{s}}$ el coeficiente de permeabilidad (o conductividad hidráulica a saturación) del medio poroso, $\left(\boldsymbol{H}_{1}-\boldsymbol{H}_{2}\right)$ la diferencia de carga (o potencial hidráulico) existente entre los lugares considerados (puntos 1 y 2) (en m), y, $\boldsymbol{e}$, el espesor del medio (en m). 
La explicación y el significado de todos los términos de la ley de Darcy se visualizan en el permeámetro de la Fig. 2.

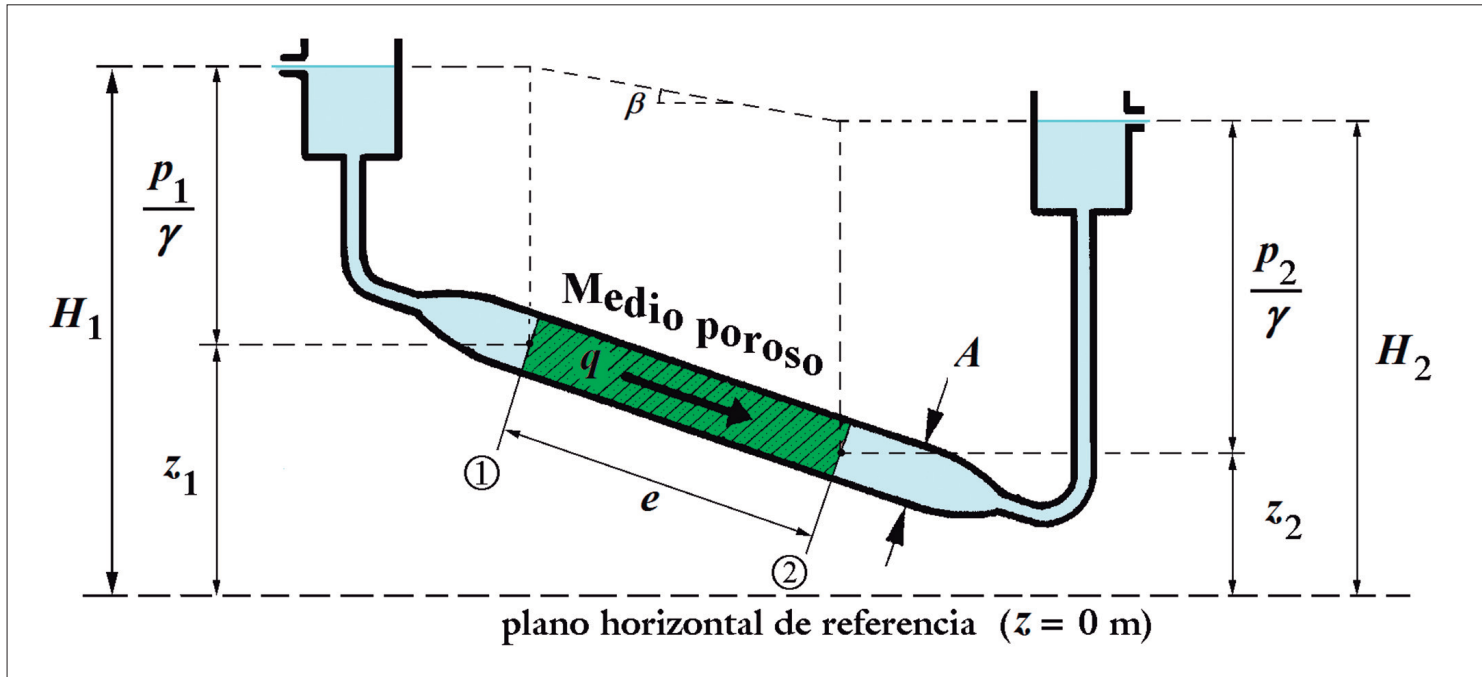

Figura 2. Explicación a la ley de Darcy a través de la representación de un permeámetro.

Al sustituir mentalmente el permeámetro por un rezumador, el depósito superior se corresponde con la botella que incorpora al rezumador y el depósito inferior con la cara exterior del rezumador. En los cálculos vamos a suponer que el rezumador tiene una permeabilidad uniforme y un espesor constante $\left(\boldsymbol{k}_{\mathrm{s}}=\right.$ cte. y $\boldsymbol{e}=$ cte.).

$\boldsymbol{H}_{\mathbf{1}}=0 \mathrm{~m}$ (como plano de referencia elegimos la superficie libre del agua en la botella de riego,

$$
\left.\boldsymbol{H}_{\mathbf{1}}=\boldsymbol{z}_{1}+\frac{\boldsymbol{p}_{\mathbf{1}}}{\rho \cdot \boldsymbol{g}}=0 ; \boldsymbol{p}_{\mathbf{1}}=\text { presión hidrostática relativa en } \mathbf{1}\right)
$$

$\boldsymbol{H}_{2}=-\boldsymbol{z}$ (potencial hídrico del agua rezumada en un punto genérico de la superficie exterior del rezumador, si éste entrega agua al aire). $\boldsymbol{H}_{2}=-\psi-\boldsymbol{z}$, si el rezumador entrega agua a un suelo que está a un potencial hídrico de $-\psi \mathrm{m}$ $\boldsymbol{k}_{\mathrm{s}}=$ Coeficiente de permeabilidad de las paredes porosas del rezumador, con $\boldsymbol{k}_{\mathrm{s}}<\boldsymbol{k}_{\mathrm{sm}}$, siendo $\boldsymbol{k}_{\mathrm{sm}}$ el coeficiente de permeabilidad del medio en donde resuda el emisor (p. ej.: el suelo)

$\boldsymbol{e}=$ espesor de las paredes del rezumador

$\boldsymbol{k}_{\mathrm{s}} / \boldsymbol{e}=$ permeabilidad unitaria (por unidad de longitud) $\left(\mathrm{en} \mathrm{s}^{-1}\right)$

$\boldsymbol{d} \boldsymbol{q}=$ caudal de resudado en una franja horizontal de superficie $\boldsymbol{d} \boldsymbol{A}$ del rezumador $\boldsymbol{q}=$ Tasa total de rezume del emisor de agua (del rezumador); ecuación característica

$$
\left(\boldsymbol{q}=\int_{A} \boldsymbol{d q}\right)
$$

$\gamma=\rho \cdot g=$ peso específico del agua $\approx 9810 \mathrm{~N} \cdot \mathrm{m}^{-3}$

En primer lugar, obtengamos el caudal resudado (o emitido) a través de una 
superficie porosa plana $(\boldsymbol{A})$ de forma cualquiera, si está rezumando al aire (Fig. 3). Posteriormente, consideraremos un caso en el que el resudado se produzca directamente al suelo.

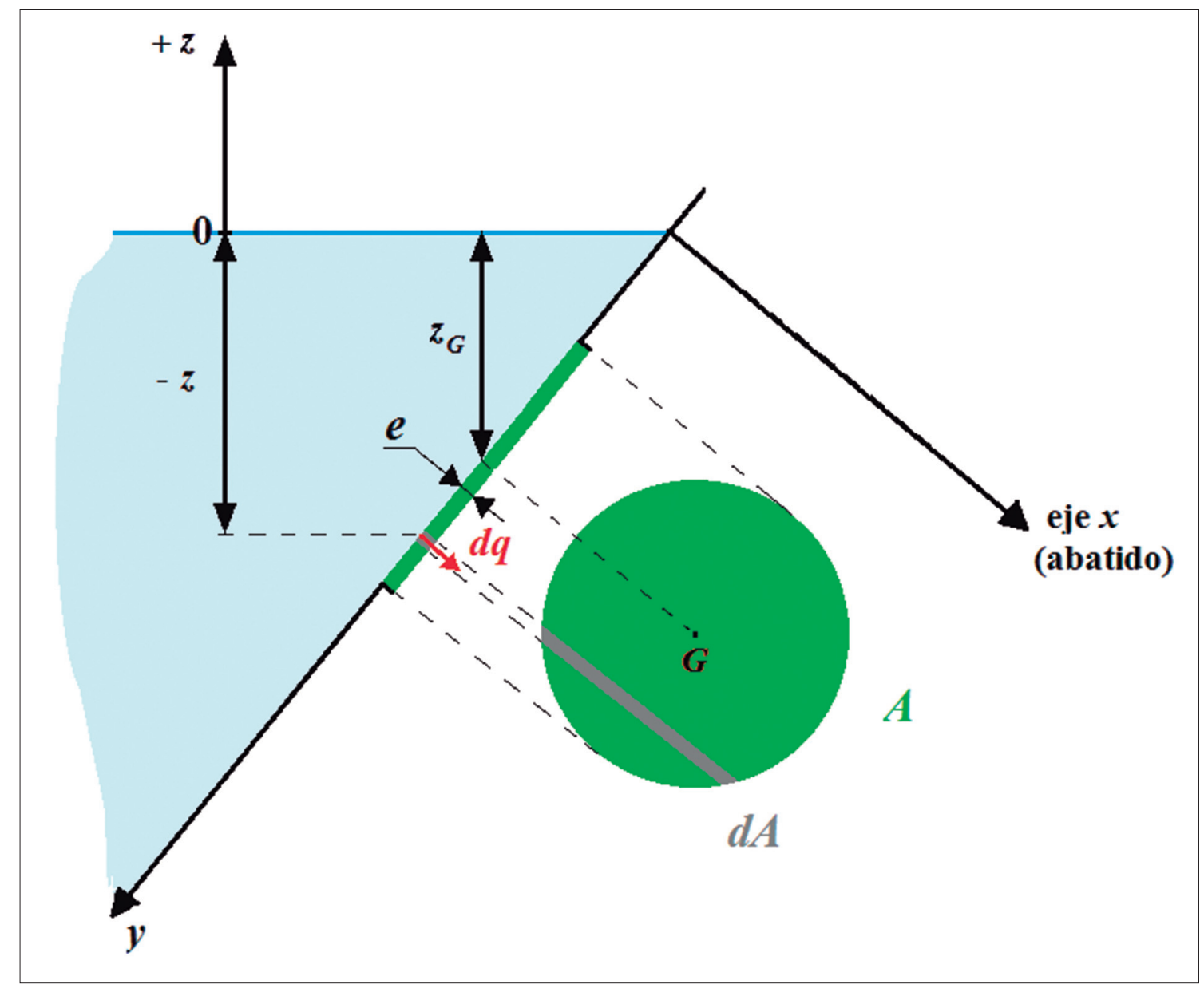

Figura 3. Esquema para hallar el caudal de rezume a través de una superficie porosa y plana con forma genérica (en el dibujo es una superficie circular de área $\boldsymbol{A}$ ).

La velocidad de flujo en el medio poroso (v) vale, conforme a la ecuación de Darcy:

$$
\boldsymbol{v}=\boldsymbol{k}_{\mathrm{s}} \cdot \frac{z}{\boldsymbol{e}}
$$

Considerando la ecuación de continuidad, el caudal volumétrico diferencial es:

Al integrar, nos queda:

$$
d q=v \cdot d A=k \cdot \cdot \frac{z}{e} \cdot d A
$$

$$
q=\int_{A} d q=\int_{A} k_{s} \cdot \frac{z}{e} \cdot d A=\frac{k_{s}}{e} \cdot \int_{A} z \cdot d A=\frac{k_{s}}{e} \cdot z_{c} \cdot A
$$

en donde $z_{\sigma}$ es la coordenada según el eje $z$ del centro de gravedad $(\boldsymbol{G})$ de la superfi- 
cie $(\boldsymbol{A})$, cuya expresión es:

$$
\begin{aligned}
\boldsymbol{z}_{\sigma}= & \frac{\int_{A^{*}}{ }^{*} d A}{\boldsymbol{A}} \\
& \boldsymbol{q}=\frac{\boldsymbol{k}_{s}}{\boldsymbol{e}} \cdot \boldsymbol{z}_{\sigma} \cdot \boldsymbol{A}=\boldsymbol{v}_{G} \cdot \boldsymbol{A}
\end{aligned}
$$

siendo $\boldsymbol{v}_{G}$ la velocidad de flujo en el centro de gravedad de la superficie porosa plana.

Conviene afectar a la fórmula deducida de un coeficiente de descarga (c) para dotar a la expresión de plena validez y posibilitar ajustes experimentales.

$$
\boldsymbol{q}=\boldsymbol{c} \cdot \frac{\boldsymbol{k}_{\mathrm{s}}}{\boldsymbol{e}} \cdot \boldsymbol{z}_{G} \cdot \boldsymbol{A}=\boldsymbol{c} \cdot \boldsymbol{v}_{G} \cdot \boldsymbol{A}
$$

Queremos señalar que esta ecuación es muy similar a la fórmula que calcula el empuje hidrostático $(\boldsymbol{E})$ sobre una superficie plana. Esta ecuación se escribe:

$\boldsymbol{E}=\rho \cdot \boldsymbol{g} \cdot \boldsymbol{z}_{G} \cdot \boldsymbol{A}=\boldsymbol{p}_{G} \cdot \boldsymbol{A} ;$ de manera que la correspondencia es: $\left.\rho \cdot \boldsymbol{g} \leftrightarrow \frac{\boldsymbol{k}_{s}}{\boldsymbol{e}}\right)$.

Si el resudado de la pared porosa (es decir: del rezumador) no se produce al aire (a la atmósfera) sino que se produce dentro de un suelo que se encuentra a un potencial hídrico de $-\psi \mathrm{m}$, y siempre que el contacto entre el rezumador y el suelo sea pleno, las ecuaciones a considerar son:

$$
\begin{gathered}
\boldsymbol{v}=\boldsymbol{k}_{s} \cdot \frac{z+\psi}{e} \\
\boldsymbol{d q}=\boldsymbol{v} \cdot \boldsymbol{d A}=\boldsymbol{k}_{s} \cdot \frac{z+\psi}{\boldsymbol{e}} \cdot \boldsymbol{d A}
\end{gathered}
$$

Finalmente:

$$
\boldsymbol{q}=\boldsymbol{c} \cdot \frac{\boldsymbol{k}_{\mathrm{s}}}{\boldsymbol{e}} \cdot\left(\boldsymbol{z}_{G}+\psi\right) \cdot \boldsymbol{A}
$$

Esta última ecuación [3] estima el caudal emitido por un rezumador plano en un suelo que esté a un potencial hídrico de $-\psi \mathrm{m}$.

Siempre que el potencial hídrico negativo sea importante con relación a la coordenada $\boldsymbol{z}_{G}$, se puede prescindir de este sumando $\boldsymbol{z}_{G}$, de manera que:

$$
\boldsymbol{q} \approx \boldsymbol{c} \cdot \frac{\boldsymbol{k}_{s}}{\boldsymbol{e}} \cdot \psi \cdot \boldsymbol{A}
$$

De acuerdo con la ecuación [4] el rezumador proporciona un caudal que es proporcional al potencial hídrico existente en el suelo. De ahí el término de riego por succión (Bordas et Mathieu, 1930; Olguín, 1975): el suelo toma agua del emisor según la va necesitando, según sea su potencial hídrico, que a su vez depende de la traspiración de las plantas.

Todo el circuito hidráulico, desde la botella, pasando por el rezumador, hasta las raíces del brinzal, está interrelacionado. Al flujo saturado en el rezumador le sigue uno insaturado a través del suelo, regido por la ecuación de Buckingham - Darcy (1907). En todo este recorrido (recipiente - raíz) podemos considerar tres potenciales carac- 
terísticos distintos (Fig. 4): el potencial en el recipiente $\left(\psi_{1} \approx 0 \mathrm{~m}\right)$, el potencial a la salida del rezumador ( $\psi_{2}$ o potencial hídrico de diseño) y el potencial junto a las raíces $\left(\psi_{3}\right.$, adecuado para que el brinzal se establezca).

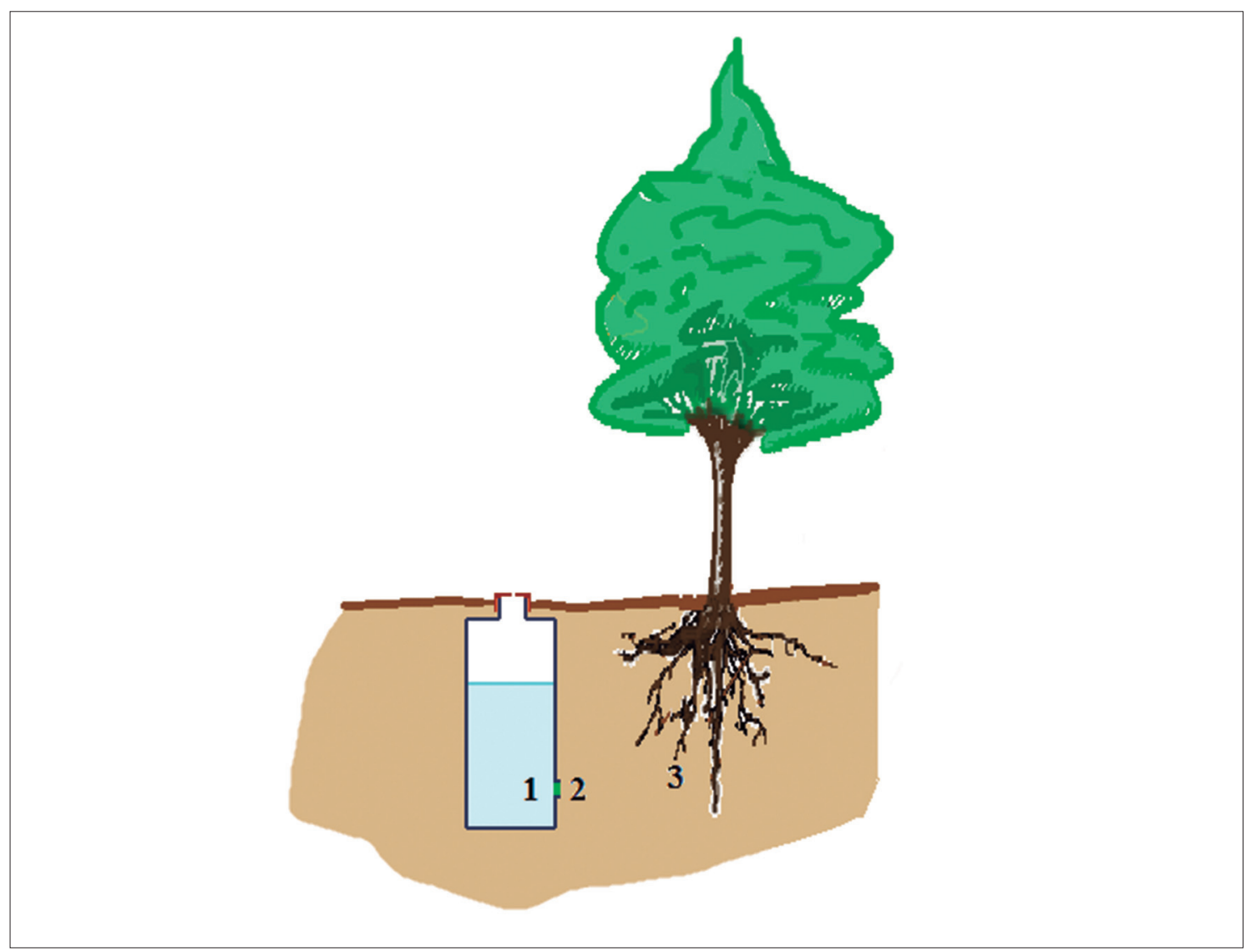

Figura 4. Gradiente de potencial hídrico entre el biberón y el brinzal.

Para un coeficiente de descarga unitario (ó de valor conocido), la ecuación [4] sirve para dimensionar el rezumador. En efecto: Sea $\boldsymbol{V}$ la capacidad de la botella de agua (del emisor de riego por succión), $\boldsymbol{k}$ s la permeabilidad del material poroso del rezumador, $\boldsymbol{A}$ su área, y $-\psi$ el potencial hídrico objetivo (o de diseño) bajo el cual se desea que la botella de riego se vacíe en un tiempo $\boldsymbol{T}$. El espesor del material poroso $(\boldsymbol{e})$ se obtiene de la ecuación siguiente:

$$
\boldsymbol{q} \cdot \boldsymbol{T}=\boldsymbol{V} \approx \frac{\boldsymbol{k}_{s}}{\boldsymbol{e}} \cdot \psi \cdot \boldsymbol{A} \cdot \boldsymbol{T} \Rightarrow \boldsymbol{e} \approx \frac{\boldsymbol{k}_{s} \cdot \psi \cdot \boldsymbol{A} \cdot \boldsymbol{T}}{\boldsymbol{V}} \quad[\text { [ec. 5] }
$$

El potencial hídrico $(-\psi)$ debe estar comprendido entre la capacidad de campo (aproximadamente: $-3 \mathrm{~m}$ ) y el punto de marchitez permanente (aprox. $-150 \mathrm{~m}$ ). Cuanto más pequeño tomemos el potencial hídrico objetivo, el riego por succión será más deficitario. Tal puede ser el caso en los riegos de socorro y de supervivencia aplicados a brinzales xerofíticos. 


$$
\psi \in[-\mathbf{1 5 0} ;-\mathbf{3}]
$$

Con un criterio edáfico y fisiológico, el potencial hídrico objetivo $(-\psi)$ puede fijarse a partir de las curvas características de humedad del suelo a regar, imponiendo que el rezume de diseño del emisor $(\boldsymbol{q})$ se produzca con un potencial que implique un contenido volumétrico de humedad $(\theta)$ concreto. Como caudal de diseño del rezumador $(\boldsymbol{q})$ puede fijarse uno que garantice un riego continuado durante el tiempo $(\boldsymbol{T})$ deseado. Por poner cifras que ayuden a fijar ideas: $\boldsymbol{q}=4 \mathrm{~L} \cdot \mathrm{mes}^{-1}$ en una botella de $2 \mathrm{~L}$, para que esté humectando el suelo durante 2 semanas antes de vaciarse.

En el caso de que el contacto entre el rezumador y el suelo sea deficiente (incompleto), la entrega de agua puede ser bastante inferior a la obtenida por la ecuación [3]. Como situación extrema puede ocurrir que el contacto sea nulo, es decir: que haya una cavidad de aire intercalada entre el rezumador y el suelo. En tal caso, el caudal rezumado se regirá por la ecuación [2]. Por tanto, podemos afirmar que el caudal emitido por un rezumador estará comprendido entre esos dos valores extremos:

$$
\boldsymbol{q} \in\left[\boldsymbol{c} \cdot \frac{\boldsymbol{k}_{s}}{\boldsymbol{e}} \cdot \boldsymbol{z}_{G} \cdot \boldsymbol{A} ; \boldsymbol{c} \cdot \frac{\boldsymbol{k}_{s}}{\boldsymbol{e}} \cdot\left(\boldsymbol{z}_{G}+\psi\right) \cdot \boldsymbol{A}\right]
$$

Estando el rezumador bien instalado (es decir: en contacto íntimo con el suelo) emitirá el valor superior, pero si queda una cámara de aire interpuesta entre el emisor y el suelo regirá la ecuación [2].

Por último, señalar que si el sistema de riego funciona a presión, puede remplazarse el término $\boldsymbol{z}_{G}$ por la presión de trabajo ( $\boldsymbol{h}$, en metros de columna de agua) existente en el centro de gravedad de la pared del rezumador, es decir:

$$
\boldsymbol{q} \in\left[\boldsymbol{c} \cdot \frac{\boldsymbol{k}_{s}}{\boldsymbol{e}} \cdot \boldsymbol{h} \cdot \boldsymbol{A} ; \boldsymbol{c} \cdot \frac{\boldsymbol{k}_{s}}{\boldsymbol{e}} \cdot(\boldsymbol{h}+\psi) \cdot \boldsymbol{A}\right] \quad \text { [ec. 7] }
$$

El tamaño del intervalo de caudales acotado por la expresión [7] depende del potencial hídrico de diseño $(-\psi)$. Cuanto más bajo (o negativo) sea, mayor será el intervalo. Por el contrario, si el potencial hídrico de diseño se elige alto (próximo a la capacidad de campo) y los rezumadores funcionan a presión, el intervalo será pequeño, lo que asegura riegos uniformes.

Debemos insistir en que para brinzales xerofíticos trabajar con presión es un gasto superfluo, pues supone desaprovechar una de las importantes ventajas que posee el sistema: poder funcionar a presión nula. Si se desea más caudal de riego, basta con elegir un rezumador más grande (de mayor superficie o de menor espesor de pared, o más rezumadores por botella, por planta a regar), en vez de dar presión a una red de tuberías con el consiguiente gasto energético.

\section{Algunas consideraciones prácticas}

El riego por succión a través de rezumadores tiene una tasa de entrega que depende de la sequedad del suelo ( $\psi$; ecuaciones [3] y [4]). Ello permite un gran ahorro de 
agua pero conduce a caudales de riego muy variables de unos lugares a otros dentro de la misma repoblación. Esta característica supone un inconveniente en riegos agrícolas de producción (evitable con la presión $\boldsymbol{h}$ ) pero no afecta a la finalidad última de un micro-riego de apoyo a brinzales. Más bien al contrario, se trata de una ventaja sustancial en riegos de establecimiento y supervivencia de especies xerófitas, cuando la fuente de abastecimiento de agua sea escasa. Como los descensos tolerables de humedad pueden ser muy grandes en xerófitas sin que se produzcan marras por estrés hídrico, la uniformidad del riego pasa a un segundo plano mientras que el ahorro de agua resulta prioritario: dosis de riego mínimas y máxima eficiencia en la aplicación del agua.

Si el contacto entre el emisor y el suelo no es íntimo, el caudal puede ser bastante menor (por rezumar el emisor al aire; intervalo definido en las expresiones [6] y [7]). Otro hecho constatado es que, con el paso de los años, las raíces terminan por forrar al rezumador con una tupida borra de raicillas (hidrotropismo), lo que también suele reducir el caudal entregado al hacerlo el potencial hídrico $(\psi)$. Pero como los riegos de brinzales son riegos temporales, que se practican durante el primer año o durante unos pocos años, hasta que la vegetación introducida alcanza su autonomía hídrica, este efecto tiene muy escasa relevancia. Comentario similar cabe hacer respecto a la posibilidad de que las raíces penetren en el interior del recipiente a través del propio rezumador. Este riesgo es más probable con raíces agresivas, que son muy activas en la búsqueda de agua: en especies freatófilas y no en brinzales xerófilos.

La pared de un rezumador es sensible a las obturaciones pero ello no interrumpe su resudado, sino que lo disminuye, pues el agua dispone de muchos recorridos hidráulicos alternativos cuando el medio poroso es amplio ( $\boldsymbol{A}$ grande). Lógicamente y si se utilizan aguas turbias o con muchos microorganismos, la reducción de $\boldsymbol{k}$ s con el paso de los meses es ostensible. A su vez y en climas con fuertes heladas, éstas pueden alterar la porosidad del rezumador de un año al siguiente, provocando microgrietas que aumentan $\boldsymbol{k}$.

\section{Conclusiones}

- El microrriego a través de recipientes enterrados con rezumadores (superficies porosas) tiene mucho interés para el establecimiento de brinzales en zonas áridas y en áreas críticas por acomodarse a riegos deficitarios y por su alta eficiencia en la aplicación del agua.

- La ecuación característica de un rezumador se obtiene por integración a partir de la ecuación de Darcy y la ecuación de continuidad, concretando las condiciones de contorno.

- Estas condiciones de frontera son bastante diferentes según que el emisor rezume al aire o directamente al suelo (¡succión!) lo que se traduce en un intervalo de caudales amplio.

- En microrriegos forestales, con especies xerófilas y frugales, estas diferencias en la entrega de caudales tienen poca importancia (siempre que estén bien aco- 
tadas), pues se trata de riegos de supervivencia. No son riegos de producción.

- Para realizar el diseño técnico de un riego a través de rezumadores hay que conocer la ecuación característica de estos emisores, hay que fijar un potencial hídrico objetivo y hay que determinar el volumen de los recipientes, de manera que se vacíen en el tiempo deseado.

- La industria actual del plástico y de sus derivados posibilita la obtención de nuevos materiales porosos con los que fabricar rezumadores. Ello augura un amplio desarrollo del riego por succión en el futuro.

\section{Agradecimientos}

Este trabajo se enmarca dentro del proyecto LIFE 09/ENV/ES/447: "Los desiertos verdes: nuevas técnicas de plantación de árboles en ambientes desertizados para afrontar el cambio climático".

\section{Bibliografía}

Bainbridge, D. A., 2001. Buried clay pot irrigation: a little known but very efficient traditional method of irrigation. Agricultural Water Management, 48: 79-88.

Bainbridge, D. A., 2002. Alternative irrigation systems for arid land restoration. Ecological Restoration, 20 (1): 23-30.

Bainbridge, D. A., 2007. A guide for desert and dryland restoration. New hope for arid lands. Island Press. Washington.

Bordas, J., Mathieu, G., 1930. Récherches sur la force de succion des sols et l'irrigation souterraine. Annales de la Science Agronomique, 47: 192-235.

Buckingham, E., 1907. Studies on the movement of soil moisture. Bulletin 38. USDA Bureau of Soils, Washington, DC.

Darcy, H., 1856. Les fontaines publiques de la Ville de Dijon. Editorial V. Dalmont. París.

Martínez de Azagra, A., Del Río, J., 2012. Los riegos de apoyo y de socorro en repoblaciones forestales. Foresta, 53: 32-44.

Matorel García, M., 1996. Programa piloto de reforestación extensiva. Bosques para el futuro. Documento inédito. Universidad de Piura (Perú).

Matorel García, M., 1998. Riego en el desierto. Reservorios de exudación subterránea. Revista Agronoticias, 223: 34 (Lima, Perú).

Mondal, R.C., 1974a. Farming with pitcher: a technique of water conservation. World Crops, 26 (2): 91-97.

Mondal, R.C., 1974b. Pitcher farming: a simple low-cost method of irrigation. Appropriate Technologies, 13: 7-8.

Navarro Hevia, J.; Martínez de Azagra Paredes, A., Mongil Manso, J. (eds.), 2009. Hidrología de Conservación de Aguas. Captación de precipitaciones horizontales y escorrentías en zonas secas. Edita Universidad de Valladolid. Valladolid.

Olguín Palacios, C., 1975. Riego por succión. Descripción del método y avances en la in- 
vestigación. In: Memorias del $1^{\text {er }}$ Seminario Nacional de Riego por Goteo (tomo II: 165-181). Hermosillo. Sonora. México.

Olguín Palacios, C., 1977. Observaciones sobre el efecto de riego por succión en el rendimiento y desarrollo de maíz (variedad $H$-507) en el distrito de riego $N^{o}$. 41, Río Yaqui. Sonora. Memorándum Técnico, 364. SARH, México: 18 pp.

Ortega Sastriques, F., Forbes López, T., De la Rosa, S., 1986. Uso de potes porosos en la repoblación forestal, en suelos de zonas secas. Ciencias de la Agricultura, 27: 159-160 con 2 láminas.

Serrada Hierro, R., Navarro Cerrillo, R.M., Pemán García, J., 2005. La calidad de las repoblaciones forestales: una aproximación desde la selvicultura y la ecofisiología. Investigación Agraria: Sistemas y Recursos Forestales, 14 (3): 462-481.

Sheng-Han, S., 1974. On "Fan Shêng-chih Shu": An Agriculturist Book of China written by Fan Shêng-chih Shu in the First Century BC. Science Book. Peking. pp.: 36-37.

Sheikh, M.T., Shah, B. H., 1983. Establishment of vegetation with pitcher irrigation. Pakistan Journal of Forestry, 33(2): 75-81.

Stein, T.-M., 1994. Grundlagen und Technik der Gefäßbewässerung. - Zeitschrift für Bewässerungswirtschaft, 29 (1): 62-94.

Stein, T.-M., 1998. Erarbeitung und Überprüfung von Entwurfskriterien für Gefäßbewässerungsanlagen. Der Tropenlandwirt (Journal of Agriculture in the Tropics and Subtropics), 66: 1-175.

Tilló Torres, J., 2006. Riego por exudación. Ponencia al Congreso Internacional ApliMatec 06 (Aplicaciones Técnicas de los Materiales Textiles). Valencia.

UNEP, 1997. Sourcebook of alternative technologies for freshwater augmentation in Latin America and the Caribbean. Chapters 4.2 y 5.7 (sobre cápsulas porosas). UNEP / OAS. Washington. http://www.greenstone.org/ Accessed. 26 November 2014. 
